\title{
Analysis of nanoparticles optical propagation influence in biological tissue simulating phantoms
}

\author{
Miguel A. Rodríguez-Colmenares, Félix Fanjul-Vélez*, Laura Arévalo-Díaz, José L. Arce-Diego* \\ Applied Optical Techniques Group, TEISA Department, University of Cantabria, Av de los Castros \\ s/n, 39005, Santander (Spain)
}

\begin{abstract}
The applications of nanoparticles in optical techniques of diagnosis and treatment of biological tissues are increasing. Image contrast can be improved in diagnostic approaches such as fluorescence, spectroscopy or optical coherence tomography. The therapeutic effect can be increased if nanoparticles are previously incorporated in the biological tissue. This is the case in thermotherapy, or in Photodynamic Therapy. All these applications take advantage of specific properties of the nanoparticles involved, either optical up- or down-conversion, thermal confinement or the ability to act as a drug-carrier.

Although many biomedical applications that involve nanoparticles are being proposed and tested, there is a need to take into account the influence of those nanoparticles on optical radiation propagation. The previously mentioned optical treatment and diagnosis techniques assume a particular optical propagation pattern, which is altered by the addition of nanoparticles. This change depends on the nanoparticle material, shape, size and concentration, among other parameters. In order to try to quantify these changes, in this work several phantoms that include different nanoparticles are analyzed, in order to estimate the influence of nanoparticles in optical propagation. A theoretical model of optical propagation, which takes into account the absorption and scattering changes in the medium, is also considered. Nanoparticles of different sizes from $40 \mathrm{~nm}$ to $1 \mu \mathrm{m}$ are analyzed. Nanoparticle materials of interest in biomedical applications are employed. The results are relevant in diagnosis interpretation of images and treatment outcome evaluation when nanoparticles are present.
\end{abstract}

Keywords: nanoparticles, tissue simulating phantoms, optical propagation, optical properties

\section{INTRODUCTION}

The use of bio-nanotechnology is a promising novel strategy for the development of new ways to overcome actual drawbacks in clinical applications [1]. The combination of different types of nanoparticles with current therapeutic techniques offers new possibilities to biomedicine, such as improved drug carriers, energy transducers to absorb the light and transfer the energy to the drug molecule or even photodynamically active nanoparticles, as in the case of photodynamic therapy [2]. They can even lead to a selective localization of hyperthermic treatment due to their capacity of being targeted to the tumor in vivo and subjected to laser irradiation from an external source [3]. Nanoparticles have also an important role in the field of diagnosis, offering a solution to improve the disadvantages of conventional agents, such as poor contrast, photobleaching, and low chemical and optical stability in a biological environment [4]. During the last years, a big effort had been devoted to the research of different types of nanostructures, synthesis and manufacturing methods, new biomedical applications and the study of the optical and thermal effects in the medium surrounding them [5]. The use of models that take into account those effects has become an important tool in order to predict the response of biological environments when nanoparticles are administered in the target tissue.

Optical phantoms are quite relevant in biomedical optical techniques applications, as they offer the possibility to test a priori assumptions by mimicking optical properties of biological tissues [6]. Optical phantoms can be built based on several materials, such as homogenized milk, non-dairy creamer, wax, or even suspensions of oils/fats in an aqueous solution such as Intralipid and India ink. The previously mentioned materials do not allow for multilayered structures, and present difficulties in building inhomogeneities. In order to cope with these issues, phantoms can be made from materials such as silicone, polyester, polyurethane or epoxy resin.

*fanjulf@unican.es; arcedj@unican.es; phone+34 942 201545; fax +34 942 201873; www.teisa.unican.es/toa

Optical Interactions with Tissue and Cells XXVIII, edited by E. Duco Jansen, Hope Thomas Beier, Proc. of SPIE Vol. 10062, 100620W · @ 2017 SPIE · CCC code: 1605-7422/17/\$18 · doi: 10.1117/12.2252452 
In this work optical phantoms are employed in order to analyze the influence of several types of nanoparticles in optical radiation propagation. Nanoparticles parameters, such as material type or size, influence optical radiation distribution. The adequate consideration of optical propagation is essential to an accurate a priori knowledge of treatment or diagnosis optical techniques results. In section 2 the theoretical model of nanoparticles optical properties and optical radiation propagation is presented. Section 3 contains results on specific nanoparticles optical properties, optical radiation influence and phantoms. Some conclusions are presented in section 4.

\section{NANOPARTICLES AND OPTICAL RADIATION MODEL}

Optical radiation propagation can be modeled by means of different approaches. For the present problem the distribution of light in a three-dimensional tissue must be obtained. This objective is reached by means of the Radiation Transport Theory (RTT) [7]. The model assumes that the scattering events are sufficiently numerous as to the light to be considered incoherent, so polarization or interference effects can be neglected. As a consequence, the basic parameter of light is the specific intensity $I(r, \hat{s})$, that is, the light power per unit area per unit solid angle. The radiation is expected to be at the point $\vec{r}$, and to follow the direction $\hat{s}$. The scattering events are treated according to the scattering phase function $p\left(\hat{s} \cdot \hat{s}^{\prime}\right)$, which contains the probabilities of light to be scattered in the different directions. Optical radiation comes from direction $\hat{s}^{\prime}$ and is redirected to $\hat{s}$. The basic idea in order to write the differential radiation transport equation is that radiation from a particle attenuates due to absorption and scattering and also gains power because another particle can scatter light in the direction of the particle of interest. This can be written:

$$
\hat{s} \cdot \bar{\nabla} I(r, \hat{s})=-\left(\mu_{a}+\mu_{s}\right) I(r, \hat{s})+\frac{\mu_{s}}{4 \pi} \int_{4 \pi} p\left(\hat{s} \cdot \hat{s}^{\prime}\right) I\left(r, \hat{s}^{\prime}\right) d \Omega^{\prime}
$$

The absorption and scattering events which optical radiation suffers as it propagates through the biological medium with embedded nanoparticles must take into account contributions from optical properties depending on the type of pathological tissue and from nanoparticles. In order to quantify both contributions, absorption and scattering coefficients, $\mu_{\mathrm{a}}$ and $\mu_{\mathrm{s}}$ respectively, have been calculated by equations (2) and (3), where the subscript NP indicates properties of nanoparticles and the subscript $t$ indicates properties of the tissue or phantom.

$$
\begin{gathered}
\mu_{a}=\mu_{a N P}+\mu_{a t} \\
\mu_{s}=\mu_{s N P}+\mu_{s t}
\end{gathered}
$$

Numerical analysis has been widely applied to a great variety of problems governed by differential equations. In the particular topic of the radiation transport equation, the Monte Carlo method has demonstrated its applicability and accuracy, compared with exact solutions. Perhaps the most used implementation of the Monte Carlo method applied to the RTT model is the one by Wang and Jacques, $[8,9]$. This implementation of the Monte Carlo model is also multilayered, so it is possible to define several layers of different materials, with their borders always perpendicular to the laser beam, which is very useful due to tissues usually can be divided in different strata.

The optical response of tissues varies depending on the type and size of the nanoparticle embedded in the target tissue [10]. In order to get the optical absorption and scattering properties of nanospheres of arbitrary radius, the efficiency factors of absorption $\mathrm{Q}_{\mathrm{abs}}$, scattering $\mathrm{Q}_{\mathrm{sca}}$ and extinction $\mathrm{Q}_{\mathrm{ext}}$, can be calculated by means of Mie's theory for an homogeneous spherical nanoparticle with radius $\mathrm{r}$ [11].

$$
\begin{gathered}
Q_{\text {sca }}=\frac{2}{x^{2}} \sum_{n=1}^{\infty}(2 n+1)\left(\left|a_{n}\right|^{2}+\left|b_{n}\right|^{2}\right) \\
Q_{\text {ext }}=\frac{2}{x^{2}} \sum_{n=1}^{\infty}(2 n+1) \operatorname{Re}\left(a_{n}+b_{n}\right) \\
Q_{a b s}=Q_{\text {ext }}-Q_{\text {sca }} .
\end{gathered}
$$

The amplitude coefficients $a_{n}$ and $b_{n}$ for the scattered field are calculated by the expressions 7 and 8 . In these equations spherical Bessel functions are used. The parameters are refractive index of the sphere relative to the host medium $\mathrm{m}$, the size parameter $\mathrm{x}=\mathrm{kr}$, and the wave number $\mathrm{k}=2 \pi / \lambda$, where $\lambda$ is the wavelength in the ambient medium and $\mu_{1}$ is the ratio of the magnetic permeability of the sphere to the magnetic permeability of the host medium. 


$$
\begin{gathered}
a_{n}=\frac{m^{2} j_{n}(m x)\left[x j_{n}(x)\right]^{\prime}-\mu_{1} j_{n}(x)\left[m x j_{n}(m x)\right]^{\prime}}{m^{2} j_{n}(m x)\left[x h_{n}^{(1)}(x)\right]^{\prime}-\mu_{1} h_{n}^{(1)}(x)\left[m x j_{n}(m x)\right]^{\prime}} \\
b_{n}=\frac{\mu_{1} j_{n}(m x)\left[x j_{n}(x)\right]^{\prime}-j_{n}(x)\left[m x j_{n}(m x)\right]^{\prime}}{\mu_{1} j_{n}(m x)\left[x h_{n}^{(1)}(x)\right]-h_{n}^{(1)}(x)\left[m x j_{n}(m x)\right]^{\prime}}
\end{gathered}
$$

Nanoparticle absorption and scattering coefficients are quantified from efficiency parameters, cross section of the spherical nanoparticle and number of nanoparticles per unit volume by the following expressions

$$
\begin{aligned}
& \mu_{\text {abs }}=\pi r^{2} Q_{a b s} N \\
& \mu_{\text {sca }}=\pi r^{2} Q_{\text {sca }} N
\end{aligned}
$$

\section{RESULTS AND DISCUSSION}

The previously developed approach is applied to different types of nanoparticles. As an example, efficiency factors of Au spherical $80 \mathrm{~nm}$ radius nanoparticles with a uniform concentration of $2 \cdot 10^{9} \mathrm{~cm}^{-3}$ as a function of wavelength were obtained, and the results appear in Figure 1.

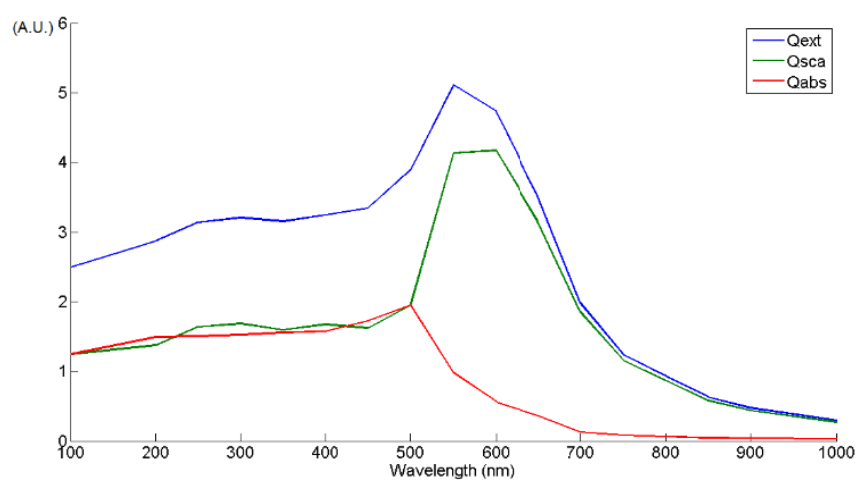

Figure 1. Efficiency factors of absorption (Qabs), scattering (Qsca) and extinction (Qext) (A.U.) of Au spherical nanoparticles.

From Figure 1 it is possible to calculate absorption and scattering coefficients, which are of great relevance in optical radiation propagation. Figure 2 shows these results.

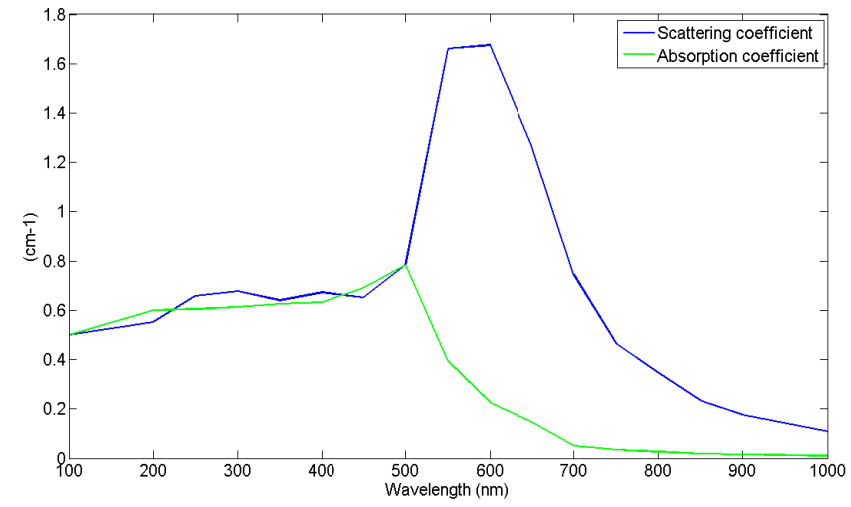

Figure 2. Scattering and absorption coefficients $\left(\mathrm{cm}^{-1}\right)$ of Au nanoparticles as a function of wavelength.

From these results it is possible to estimate optical radiation propagation in biological tissues or biological tissue phantoms. Light distribution with and without nanoparticles embedded was obtained. A top hat laser beam with a 0.25 
$\mathrm{cm}$ radius was used to deliver an irradiance of $200 \mathrm{~mW} / \mathrm{cm}^{2}$. Figure 3 shows the optical absorption in the in the host material and using $80 \mathrm{~nm}$ radius Au nanoparticles with a density of $4 \cdot 10^{9} \mathrm{~cm}^{-3}$. As it can be observed, the presence of Au nanoparticles involves greater absorption in the most superficial regions of the material.

a)

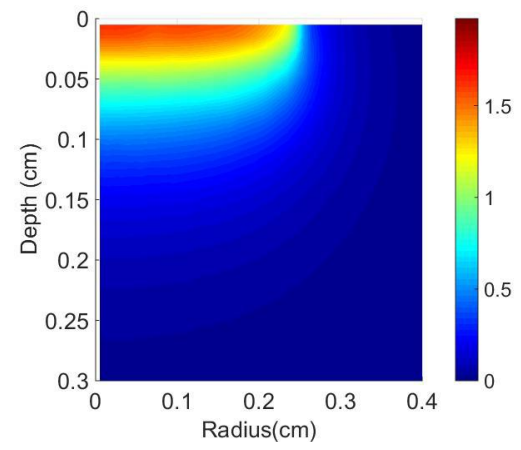

b)

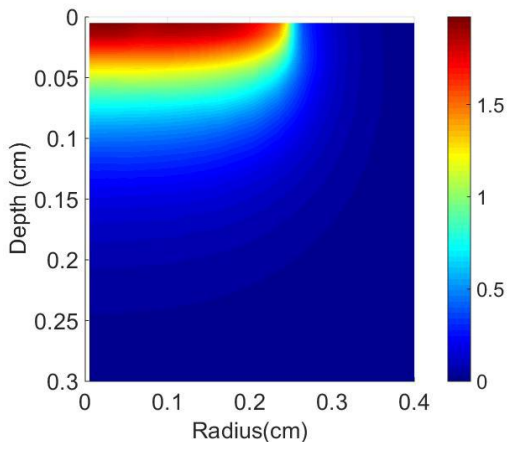

Figure 3. Optical absorption (W/cm3) in the host material without (a) and with Au nanoparticles (b).

Optical propagation with nanoparticles results in an increase of the absorption, and as a consequence in a reduction of the light penetration in the target tissue. The differences are strongly dependent on the nanoparticle size, the concentration, as well as on the wavelength of the optical radiation employed. The effective penetration depth, given by $\delta=1 / \sqrt{3 \mu_{a} \mu_{s}^{\prime}}$

according to the diffusion limit, can be calculated for different wavelengths and nanoparticles types. Table 1 contains some results. Figure 4 shows an example of the fabricated optical phantoms.

Table 1. Effective penetration depth (cm) for several spectral bands of Au nanoparticles.

\begin{tabular}{|c|c|c|c|c|}
\hline $\begin{array}{c}\lambda \\
(\mathbf{n m})\end{array}$ & $\begin{array}{c}\text { No } \\
\text { nanoparticles }\end{array}$ & $\begin{array}{c}\text { Radius }=40 \mathrm{~nm} \\
\text { Density }=2 \cdot 10^{9} \mathrm{~cm}^{-3}\end{array}$ & $\begin{array}{c}\text { Radius }=80 \mathrm{~nm} \\
\text { Density }=2 \cdot 10^{9} \mathrm{~cm}^{-3}\end{array}$ & $\begin{array}{c}\text { Radius }=80 \mathrm{~nm} \\
\text { Density }=4 \cdot 10^{9} \mathrm{~cm}^{-3}\end{array}$ \\
\hline 500 & 0.0521 & 0.0505 & 0.0483 & 0.0424 \\
\hline 550 & 0.0666 & 0.0658 & 0.0603 & 0.0551 \\
\hline 600 & 0.0939 & 0.0933 & 0.0851 & 0.0779 \\
\hline 650 & 0.1290 & 0.1283 & 0.1169 & 0.1069 \\
\hline 700 & 0.1521 & 0.1517 & 0.1445 & 0.1376 \\
\hline 750 & 0.1980 & 0.1974 & 0.1890 & 0.1808 \\
\hline 800 & 0.2635 & 0.2626 & 0.2501 & 0.2382 \\
\hline
\end{tabular}

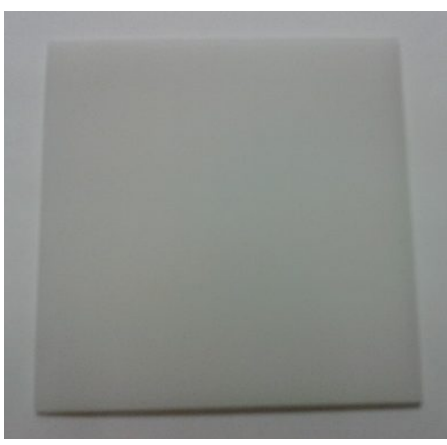

Figure 4. Optical epoxy phantom with $\mathrm{TiO}_{2}$ embedded nanoparticles. 


\section{CONCLUSIONS}

The use of nanoparticles in biomedical applications is increasing nowadays. The influence of nanoparticles in optical propagation on biological tissues can greatly affect optical distribution and, as a consequence, treatment or diagnostic efficiency. In this work a model for nanoparticles optical properties, based on Mie theory, has been presented and applied for several nanoparticles. A Monte Carlo method has been used to calculate optical propagation in host materials, either tissues or optical phantoms, with and without nanoparticles. As this type of results are difficult to obtain in real biological tissues, optical tissue mimicking phantoms are employed. The results allow to consider estimated optical radiation distribution in a particular biological tissue with particular nanoparticles and concentration.

\section{ACKNOWLEDGEMENTS}

This work has been partially supported by the project "New active phases in transition metals and rare earth nano-oxides stabilized at high pressure" (MAT2015-69508-P) of the Spanish Ministry of Economy and Competitiveness, cofunded by FEDER funds, and by the San Cándido Foundation.

\section{REFERENCES}

[1] Prasad, P. N., [Introduction to Biophotonics], John Wiley \& Sons, Inc., New Jersey (2006).

[2] Allison, R. R., Mota, B. C., Bagnato, V. S. and Sibata, C.H., "Bio-nanotechnology and photodynamic therapy: State of the art review", Photodiagnosis and Photodynamic Therapy 5, 19-28 (2008).

[3] Richardson, H. H., Carlson, M. T., Tandler, P. J., Hernández, P. and Govorov, A. O., "Experimental and Theoretical Studies of Light-to-Heat Conversion and Collective Heating Effects in Metal Nanoparticle Solutions", Nano Letters 9(3), 1139-1146 (2009).

[4] Qian, J., Fu, T., Zhan, Q. and He, S., "Using Some Nanoparticles as Contrast Agents for Optical Bioimaging", IEEE Journal of selected topics in quantum electronics 16(3), 672-684 (2010).

[5] Pustovalov, V., Astafyeva, L. and Jean, B., "Computer modeling of the optical properties and heating of spherical gold and silica-gold nanoparticles for laser combined imaging and photothermal treatment", Nanotechnology 20, 225105, 1-11 (2009).

[6] Moffitt, T., Chen, Y. C. and Prahl, S. A., "Preparation and characterization of polyurethane optical phantoms", Journal of Biomedical Optics 11, 041103 (2006).

[7] Vo-Dinh, T., [Biomedical Photonics Handbook], T. Vo-Dinh Ed., CRC Press, Boca Raton (2003).

[8] Wang, L., Jacques, S. L. and Zheng, L., "MCML - Monte Carlo modeling of light transport in multi-layered tissues", Computer methods and programs in biomedicine 47, 131-146 (1995).

[9] Wang, L., Jacques, S. L. and Zheng, L., "CONV - Convolution for responses to a finite diameter photon beam incident on multi-layered tissues", Computer methods and programs in biomedicine 54, 141-150 (1997).

[10] Lance Kelly, K., Coronado, E., Zhao, L. L. and Schatz, G. C., "The Optical Properties of Metal Nanoparticles: The Influence of Size, Shape, and Dielectric Environment", J. Phys. Chem. B 107, 668-677 (2003).

[11] Bohren, C.F. and Huffman, D.R., [Absorption and Scattering of Light by Small Particles], John Wiley, New York (1983). 